\title{
RHIZOPHORA HARRISONII. (RHIZOPHORACEAE), UN NUEVO REGISTRO PARA LAS COSTAS DE MEXICO
}

Durante un reciente viaje, enero 26 de 1981, de observación y colecta a los manglares del Estado de Chiapas, se localizaron individuos de Rhizophora harrisonii Bleech. (V. Rico-Gray, I. Espejel No. 341A, depositados en el Herbario $\mathrm{XAL}$ ) en el borde del estero, muy cerca de la Reserva Ecológica (Manglar-Zapotón) "La Encrucijada", que pertenece al Instituto de Historia Natural de Chiapas. Esta se encuentra en el municipio de Acapetahua, a los $15^{\circ} 10^{\prime}$ de latitud norte y a los $92^{\circ} 08^{\prime}$ de longitud oeste.

Al revisar las colecciones del género Rhizophora depositadas en el Herbario de la Universidad Nacional Autónoma de México (MEXU) y del Instituto Politécnico Nacional (ENCB) y, de la literatura que menciona información sobre la distribución de sus especies en América y el oeste de Africa (Breteler, 1969 y 1977; Graham, 1964; Gregory, 1958; Standley \& Williams, 1963; VázquezYáñez, 1980 a y b) se encontró que para las Costas de México sólo se reporta Rhizophora mangle L. y, que en el resto del continente y el oeste de Africa encontramos $R$. harrisonii y $R$. racemosa, además de $R$. mangle.

Al encontrar $R$. harrisonii en Chiapas, México, se amplia su distribución conocida para América, que era entre Costa Rica y Ecuador para el Pacífico (Breteler, 1977) y, Venezuela-Surinam para el Atlántico (Breteler, 1969).

Los individuos de $R$. harrisonni se encuentran compartiendo las mismas condiciones medio ambientales con los de $R$. mangle, esto es, el borde de un estero que corre a lo largo de gran parte de la costa del Estado de Chiapas. Presenta un aporte constante de agua dulce por medio de una serie de ríos que bajan de la sierra perpendciularmente a él y, de agua salada por una serie de "bocas" permanentes que lo comunican con el mar.

En general la forma y hábito de la planta son muy similares a los de $R$. mangle, encontrándose en la zona árboles de $25 \mathrm{~m}$ de altura. En lo que si difieren muy claramente, es en el número de flores por inflorescencia, ya que $R$. mangle presenta de 1 a 4 y, $R$. harrisonii un número mayor que cuatro ( 8 en nuestro caso). Breteler (1969 y 1977) menciona una serie de caracteres complementarios (Tabla 1) pero, considera al número de flores por por inflorescencia como básico y, a la vez, el más aparente.

Es importante mencionar también, que existen diferentes criterios para nombrar a las especies del género Rhizophora en América (Greogory, 1958; Hou. 1960; Salvoza, 1936) pero, consideramos que el de Breteler (1969 y 1977) es el más claro, ya que al incluir una serie de consideraciones estadísticas, ecológicas y evolutivas hace menos confuso el análisis taxonómico. Según éste criterio $R$. harrisonii es un híbrido de $R$. mangle X $R$. racemosa y, por lo tanto, presenta características de las dos especies. Las características, pueden variar dentro de un amplio rango (fig. 1) además, se ha demostrado un bajo porcentaje de fertilidad en el polen de esa especie (Breteler, 1969).

Gregory (1958), en su trabajo sobre las Rhizophoraceae de Panamá, menciona la presencia de Rhizophora samoensis (Hochr.) Salvoza a lo largo de las costas del Pacífico mexicano, pero no menciona ejemplares de respaldo; L. Paray (No.

\section{3}

Rico-Gray V. 1981. Rhizophora harrisonii. (Rhizophoraceae), un nuevo registro para las costas de México. Boletín de la Sociedad Botánica de México 41: 163-165. 
Tabla 1. Muestra las características para reconocer a las 3 especies del género Rhizophora presentes en América y el oeste de Africa (traducido de Breteler, 1969).
R. mangle
R. harrisonii
R. racemosa

INFLORESCENCIA

LARGO

PEDUNCULO

RAMIFICACION DE

LA INFLORESCENCIA

官 PEDICELO

COPA BRACTEOLAR

BOTON FLORAL

LARGO DEL

HIPOCOTILO
1 a 4 flores numerosas flores

$4-9(13) \mathrm{cm}$

$5-12 \mathrm{~cm}$

$1.7-6(10) \mathrm{cm}$

sin ramificación, o cuando más 2; laxa en águlos angostos

(5) $7-20(25) \mathrm{mm} \quad 3-11 \mathrm{~mm}$

delgada, bilabiada intermedia, generalmente bilabiada

generalmente ovado, punta aguda

ovado a ligeramente elíptico, punta aguda

$15-20(34) \mathrm{cm} \quad 11-25 \mathrm{~cm}$ 
1467 depositado en ENCR) reporta también la presencia de $R$. samoensis para el Estado de Guerrero, México, pero dado el mal estado del ejemplar, es imposible realizar corroboración alguna. A ésta especie se le considera sinónimo de $R$. mangle.

Por último, vale la pena citar dos factores que apoyan el hecho presentado en esta nota, el primero es la presencia de polen fósil proveniente de 2 ó 3 especies de Rizophora del Mioceno de Chiapas (Langenheim, et al, 1967) y, el segundo la corriente oceánica sur (Costa Rica) -norte (Golfo de Tehuántepec) que se presenta en los primeros meses del año (Tamayo, 1962); como se sabe las corrientes marinas son las responsables de la dispersión a larga distancia de los propágulos de Rhizophora.

\section{BIBLIOGRAFIA}

BRETELER, F.J. 1969. The Atlantic species of Rhizophora. Acta Bot. Neerl., 18 (3): 434-441.

BRETELER, F.J. 1977. Ameri's Pacific species of Rhizophora. Acta Bot. Neerl., 26 (3): $225-230$.

GRAHAM, S.A. S.A. 1964. The genera of Rhizophoraceae and Combretaceae in the Southeastern United States. J. Arnold Arbor., 45: 285-301.

GREGORY, D.P. 1958. Rhizophoraceae. En: Flora of Panama. Ann. Missouri Bot. Gard., 45: 136-142

HOU, D. 1960. A review of the genus Rhizophora. Blumea, 10: 625-634.

LANGENHEIM, J.H., B.L. HACKNER \& A. BARTLETT. 1967. Mangrove pollen at the depositional siete of Oligo-Miocene amber from Chiapas, México. Bot. Mus. Leafl., Harv. Uni., 21 (10): 289-324.

SALVOZA, F.M. 1936. Rhizopora. Nat. Appl. Sc. Bul. Un. Philip., 5: 179-237. STANDLEY, P.C. \& L.O. WILLIAMS. Rhizophoraceae. En: Flora of Guatemala. Fieldiana Bot., 24 (7): 263-268.

TAMAYO, J.L. 1962. Geografía General de México. Instituto Mexicano de Investigaciones Económicas. Tomo II.

VAZQUEZ-YANES, C. 1980a. Rhizophoraceae. En: Flora de Veracruz. Fasc. 12. INIREB. 8 pp.

VAZQUEZ-YANES, C. 1980b. Notas complementarias a la familia Rhizophoraceae en Veracruz. Biotica 5 (1): 15-21.

VICTOR RICO GRAY

Proyecto Flora Yucatanense

INIREB. Apdo. Postal 281

Mérida, Yucatán. México 